\title{
Short Humeral Stems in Shoulder Arthroplasty
}

\author{
Hwang Kyun Oh, Tae Kang Lim \\ Department of Orthopaedic Surgery, Eulji Hospital, Eulji University School of Medicine, Seoul, Korea
}

\begin{abstract}
Since the introduction of shoulder arthroplasty by Neer in 1974, the design of not only the glenoid component but also the humeral component used in shoulder arthroplasty has continually evolved. Changes to the design of the humeral component include a gradually disappearing proximal fin; diversified surface finishes (such as smooth, grit-blasted, and porous coating); a more contoured stem from the originally straight and cylindrical shape; and the use of press-fit uncemented fixation as opposed to cemented fixation. Despite the evolution of the humeral component for shoulder arthroplasty, however, stem-related complications are not uncommon. Examples of stem-related complications include intraoperative humeral fractures, stem loosening, periprosthetic fractures, and stress shielding. These become much more common in revision arthroplasty, where patients are associated with further complications such as surgical difficulty in extracting the humeral component, proximal metaphyseal bone loss due to stress shielding, intraoperative humeral shaft fractures, and incomplete cement removal. Physicians have made many attempts to reduce these complications by shortening the stem of the humeral component. In this review, we will discuss some of the limitations of long-stem humeral components, the feasibility of replacing them with short-stem humeral components, and the clinical outcomes associated with short-stemmed humeral components in shoulder arthroplasty.
\end{abstract}

(Clin Shoulder Elbow 2018;21(2):105-110)

Key Words: Short stem; Humeral component; Arthroplasty; Shoulder

\section{Introduction}

Since the advent of shoulder arthroplasty in 1974, designs of prostheses have continually evolved. Although early prostheses are relatively well designed and are associated with fair clinical outcomes, their variety was restricted, meaning that physicians were not able to select stem shapes and sizes personalized to the patient's anatomy. The 2 nd generation prostheses, developed in the early 90 s, show improved modularity by allowing individualized arthroplasty; a prosthetic joint could be customized to the patient's native humeral head and metaphyseal anatomy. By this time, the fixation of the humeral stems had seen a transition from cemented to uncemented fixation. In spite of this, 2nd generation prostheses were associated with poor anatomical reconstruction of the humeral head and sometimes with complications such as overstuffing. To overcome these problems, the 3rd generation prostheses were developed for a more accurate reconstruction of the neck-shaft angle through an offset humeral head and a cemented stem. The recent 4 th generation prostheses consist of shorter stems or stemless designs, which have been adopted and are currently in use by physicians.

Within this background of the development of shoulder prosthetics, the design of the stem of the humeral component specifically has changed in many aspects: the size and presence of the proximal fin; the surface finish (smoothed, grit-blasted, or porous-coated); the shape of the stem (straight and cylindrical to anatomically contoured); and the type of fixation (cemented to press-fit uncemented). Over time, the humeral stem has become shorter and shorter (Fig. 1), because past studies have suggested, for instance, that short humeral stems allow better and more stable fixation without the need for cement than long humeral stems. In particular, past research has shown that the use of short

Received May 2, 2018. Accepted May 7, 2018.

Correspondence to: Tae Kang Lim

Department of Orthopaedic Surgery, Eulji Hospital, Eulji University School of Medicine, 68 Hangeulbiseok-ro, Nowon-gu, Seoul 01830, Korea Tel: +82-2-970-8036, Fax: +82-2-973-3024, E-mail: shouldertk@gmail.com, ORCID: https://orcid.org/0000-0001-8752-3987

Review article does not need an IRB approval.

Financial support: None. Conflict of interests: None. 
stems in shoulder arthroplasty is associated with lower incidence of postoperative complications (excluding infections) such as isolated humeral component loosening; this has led physicians to question whether a humeral stem must be long for shoulder arthroplasty. Long humeral stems are associated with stem-related complications other than humeral component loosening, such as intraoperative or periprosthetic fractures and, in particular, cement-related complications when cemented fixation is used. Moreover, revision surgeries have been known to be cumbersome when long humeral stems are used. Therefore, physicians have become aware of the need for shorter humeral stems for shoulder arthroplasty and subsequently have developed several
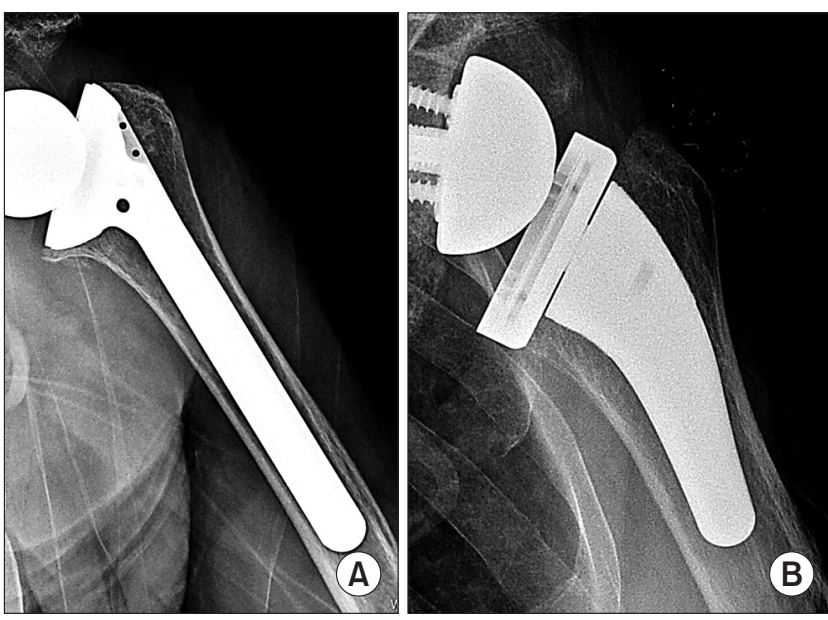

Fig. 1. Postoperative radiographs showing a standard long stem humeral component (A) and a short stem component (B) in reverse shoulder arthroplasty. prostheses with a short-stem humeral component design.

Here we classified humeral components in terms of stem length as long (>100 mm), short (<100 mm), or stemless. In this review paper, we will discuss some of the limitations of the standard long-stem humeral components and the advantages and disadvantages of short stems, by doing so deliberating the feasibility of replacing long-stemmed components with short ones. We will also compare the clinical outcomes and postoperative complications in terms of the different prosthetic implants and the type of treatment (anatomic vs. reverse arthroplasty).

\section{Limitations of Long Humeral Stems}

The clinical outcomes associated with humeral components have shown a relatively fair outcome. According to Cil et al., ${ }^{1}$ among 1,584 shoulder arthroplasties, there were only 108 rearthroplasties and 17 extractions of the humeral component. Furthermore, they found that the 10-year and 20-year survivorship of patients who underwent a shoulder arthroplasty with the humeral component were $92 \%$ and $82.8 \%$, respectively. In a systematic review on reverse shoulder arthroplasty, Zumstein et al. ${ }^{2)}$ reported that the percentage of loosening of the humeral component was lower than that of glenoid loosening $(1.5 \%$ vs. $3.5 \%)$. The relatively favorable findings for long-stem humeral components pose the question whether there is any reason for replacing the standard humeral component with the less empirically supported short-stem components. Nevertheless, there is need to pay attention to intra- or postoperative complications, as opposed to complications involving the screw itself, such as loosening, that have been associated with long-stem humeral
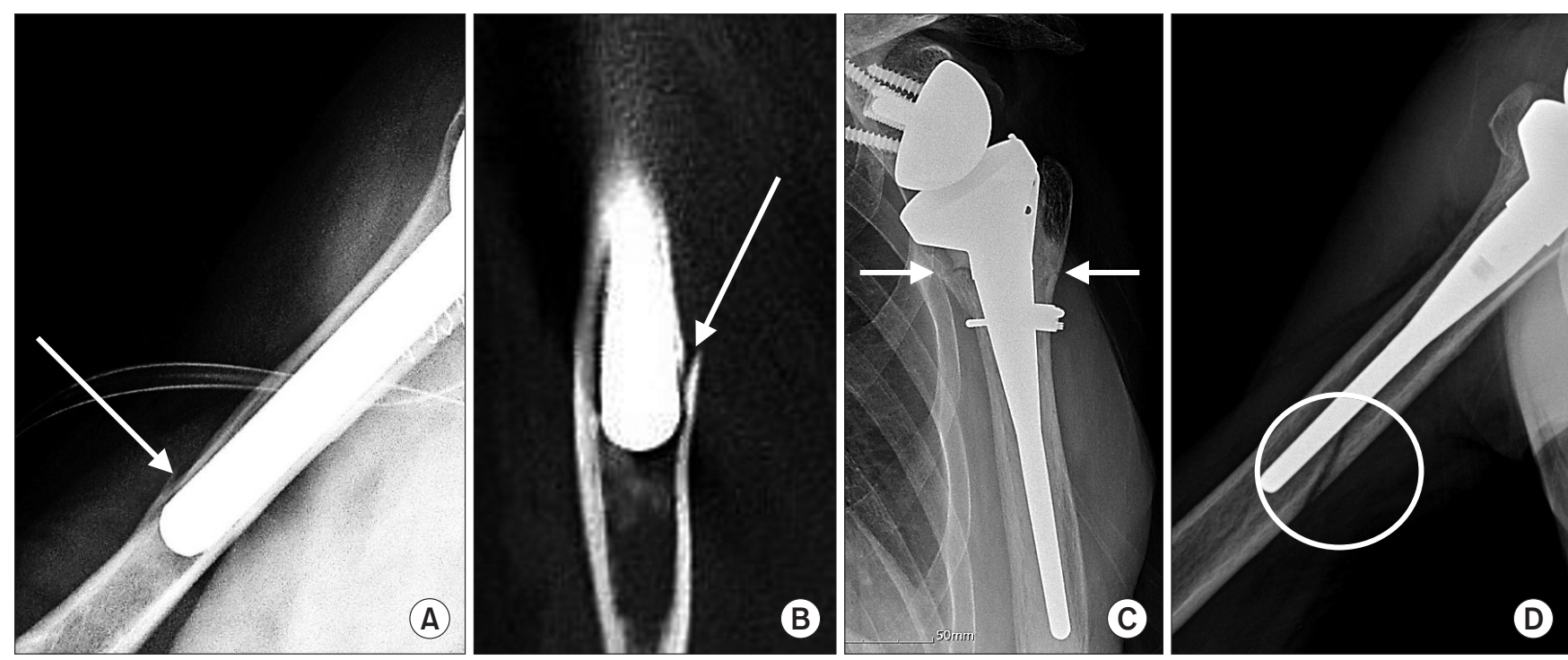

Fig. 2. Perioperative complications related to long stem humeral component, such as intraoperative humeral shaft fracture (arrows), during inserting press-fit, cementless long stem on simple $\mathrm{x}$-ray (A) and computed tomography image (B), intraoperative proximal metaphyseal fracture (arrows) that was fixed with cerclage wiring (C), and periprosthetic fracture (circle) due to simple fall after reverse shoulder arthroplasty (D). 
components.

Well-known stem-component-related complications include intraoperative humeral fractures, loosening, stress shielding, and traumatic periprosthetic humeral fractures (Fig. 2). ${ }^{3-8)}$ Zumstein et al. ${ }^{2)}$ reported that $4.2 \%$ of postoperative complications were stem-related complications, which equates to around a fifth of all postoperative complications, including periprosthetic fractures, disassembly, and loosening. Previous studies have also found that more than $70 \%$ of revision arthroplasties required the removal of the humeral component, highlighting the problem of stem-related complications for physicians.

The risk of stem-related complications is especially high for patients undergoing revision arthroplasty. Some examples of stem-related complications include surgical difficulty in extracting the humeral component, proximal bone loss due to stress shielding, osteolysis due to polyethylene wear, intraoperative fractures, and incomplete removal of cement. Cisneros et al. ${ }^{9)}$ found that in 40 patients who underwent revision shoulder arthroplasties those with stemmed implants tended to have a longer surgery, a higher likelihood to undergo humeral osteotomy and have intraoperative fractures, and showed a lower constant score than patients with stemless implants. These findings altogether suggest that stem length can play a large role in influencing the difficulty of, in particular, revision surgeries, postoperative complications, and clinical outcome. Although these are relatively rare complications, for the surgeon the occurrence of any one of them would lead to a heightened difficulty in treatment. Therefore, the need to prevent and minimize stem-related complications has led to the development of uncemented fixation approaches, through the use of press-fit stems, and to the shortening of stems.

\section{Need for Shorter Humeral Stems}

The advantages of short humeral stems, developed to overcome the limitations of long humeral stems, can be summarized as follows: (1) bone stock preservation; (2) less stress shielding; (3) no diaphyseal stress risers; (4) ease of component extraction during revision arthroplasty; and (5) a humeral-head replacement independent of the orthopedic humeral axis.

The most important advantage of short-stem humeral component is bone stock presevation, through which the risk of periprosthetic fractures, not only during the initial surgery but also potentially during the revision surgery, can be decreased. When shoulder arthroplasties using the short-stem humeral components are performed, we can see that even after metaphyseal reaming bone stock preservation of the proximal metaphysis is improved markedly (Fig. 3). In instances of periprosthetic fractures, it is often difficult to obtain a stable proximal fixation through the surrounding tissues of the humeral stem; therefore, preserving the proximal bone stock as much as possible initially can help treat potential postoperative complications that appear later on. In addition, because cortical loss occurs mostly at the tip of the stem during cases of stem erosion, stems that are longer than the original must be used during revision arthroplasty; thus, using a short stem initially can prevent these foreseeable problems. Lastly, short humeral stems can ease procedures of the revision surgery. Long-stem humeral components, especially those that have been cemented for fixation, are very difficult to remove and sometimes require a cortical window at the proximal humerus. ${ }^{10)}$ The use of short humeral stems bypasses the need to create additional cortical windows and facilities uncemented fixation, thereby lowering the difficulty of revision surgeries and the rate of surgical complications.

Second, the use of short humeral stems decreases the amount of stress shielding at the proximal humerus. Long humeral stems, however, shares the load at the diaphyseal portion of the stem, causing a relative greater stress shielding from the proximal humerus. Findings from a finite element model study investigating the relationship between humeral stem length and stress shielding show that cortical bone stress induced on the proximal humerus is $58 \%$ of the normal bone when standard long humeral stem are used. These findings show that stress shielding is more severe when long-stem humeral components are used than when short-stem (78\%) and stemless (101\%) humeral components are used. ${ }^{11)}$ Consistent with these findings, clinical studies using long humeral stems, such as those by Raiss et al., ${ }^{12)}$ have reported that the rate of radiolucency surrounding the humeral component, an indicator of the level of stress shielding, is in fact very high $(82.5 \%)$.

Third, because short humeral stems allow you to obtain

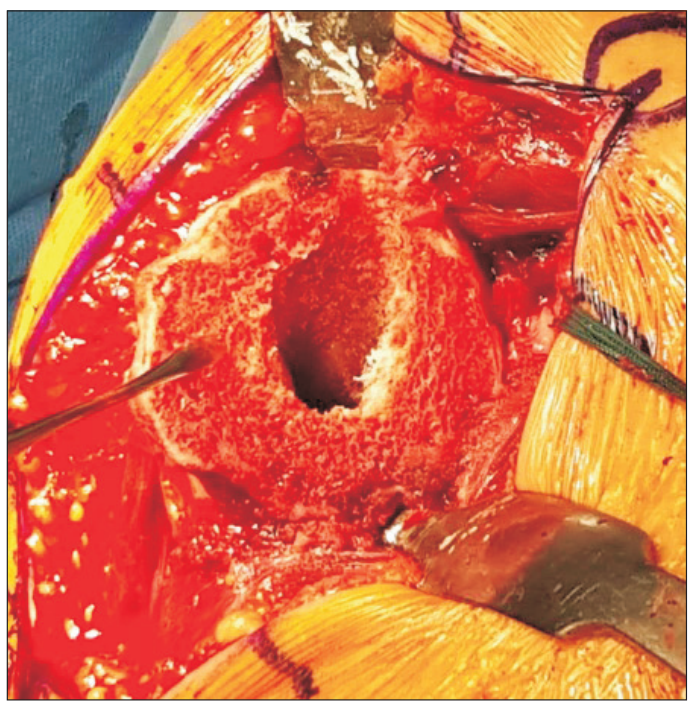

Fig. 3. Intraoperative photo of reverse shoulder arthroplasty using short humeral stem (the right shoulder in a 70-year-old female patient), showing preservation of bone stock in proximal humeral metaphysis at completion of metaphyseal reaming. 
metaphyseal stability, it can be used to decrease the incidence of diaphyseal stress risers. According to Lee et al., ${ }^{13)}$ diaphyseal reaming may lead to asymmetric reaming when long humeral stems are used, which can be a cause of stress risers in the diaphysis and therefore of periprosthetic fractures.

Lastly, the standard long-stem humeral components can be used only when the anatomic relationship between the humeral head and the humeral shaft is preserved. As a result, only patients without proximal humeral deformity and malunion, whether intrinsic or post-traumatic in origin, can be indicated for the treatment of shoulder arthroplasties using long humeral stems. In such cases, the use of short-stem or stemless humeral component may lead to better outcome.

\section{Limitations of Short Humeral Stems}

Despite potential advantages of short humeral stems that are expected to solve problems associated with long humeral stems, potential limitations of short humeral stems exist. Because long humeral stems are inserted along the medullary canal, the risk of varus or valgus misalignment of stems is lower. Compared to long humeral stems, short humeral stems have a higher risk of malalignment (Fig. 4). Moreover, suboptimal bone in-growth can lead to loosening and, thus, earlier failure when short humeral stems are used. ${ }^{14)}$ Because prosthetic implants for reverse shoulder arthroplasty have a more constrained design than, for instance, total shoulder arthroplasty, which have a more anatomic design, there is a relatively higher risk of torsional stress and micromotion, leading to an even greater risk of poor bone in-growth. ${ }^{14)}$ These limitations, which can possibly lead to complications (Table 1, 2), must be addressed through improved designs of prosthetic implants.

\section{Clinical Outcome and Postoperative Complications of Different Prosthetic Implants}

\section{1) Biomet}

Different short stem systems exist in Biomet. The Verso (Biomet, Warsaw, IN, USA) has a short metaphyseal stem design that is applicable to reverse shoulder arthroplasty. The comprehensive shoulder system consists of the Mini (83-mm-legnth stem) and the Micro, which are both commercially available in the Korean market. Atoun et al. ${ }^{15)}$ reported that the Verso humeral component was associated with an average Constant score of 56 points after at least a 24-month follow-up in 31 patients treated with shoulder arthroplasty. In another study, Jost et al. $^{16)}$ reported that the Mini humeral component was associated with an average Constant score of 91 points after at least a

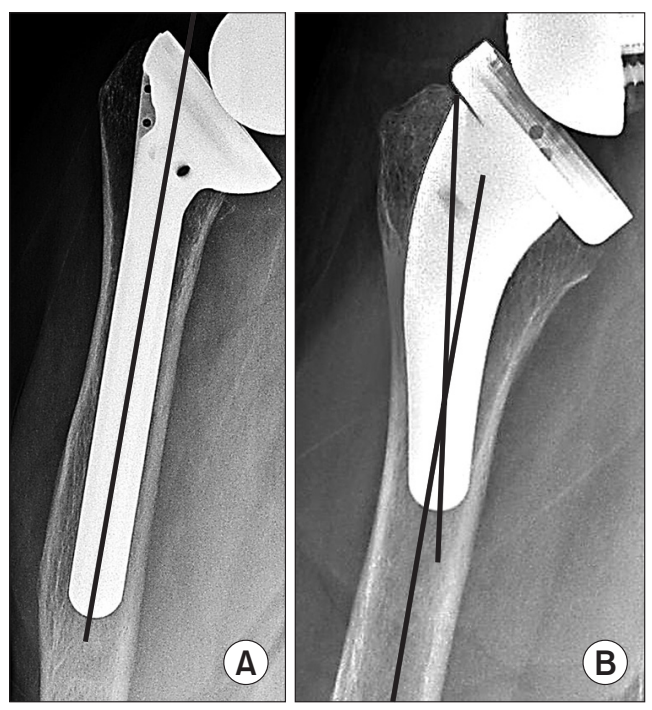

Fig. 4. Radiographs showing a well-aligned, long humeral component (A) and valgus-aligned, short humeral component (B). A long stem humeral component can be inserted well aligned, extending down the medullary canal, while a short stemmed component is prone to valgus or varus malalignment due to lack of canal-based constraint during insertion.

Table 1. Outcomes in Anatomic Total Shoulder Arthroplasty

\begin{tabular}{|c|c|c|c|c|c|c|}
\hline Study (year) & Implant & No. of patient & Age (yr) & $\mathrm{F} / \mathrm{U}(\mathrm{mo})$ & Outcome (score) & Complication \\
\hline Jost et al..$^{16)}(2011)$ & Biomet (Mini) & 47 (49 implants) & $67(46-83)$ & $29(24-43)$ & $\begin{array}{l}\text { UCLA } 27.5 \\
\text { Constant } 91\end{array}$ & $\begin{array}{l}2 \text { varus stems, } 13 \text { proximal } \\
\text { humerus resorption }\end{array}$ \\
\hline $\begin{array}{l}\text { Casagrande et al. }{ }^{17)} \\
\quad(2016)\end{array}$ & $\begin{array}{l}\text { Aequalis Ascend } \\
\text { (Tornier) }\end{array}$ & 73 implants & $64(39-86)$ & $\begin{array}{l}\text { Radiographic F/U, } 33 \\
\quad(42-50) \\
\text { Clinical F/U, } 28 \text { (24-48) }\end{array}$ & ASES 84 & $\begin{array}{l}\text { Radiolucent zone in } 71 \% \text {, } \\
\text { humeral loosening } 11 \% \text {, } \\
\text { stem revision } 8 \%\end{array}$ \\
\hline $\begin{array}{l}\text { Morwood et al. }{ }^{18)} \\
(2017)\end{array}$ & $\begin{array}{l}\text { Aequalis Ascend } \\
\text { (Tornier) }\end{array}$ & $\begin{array}{l}68 \text { (34 proximal } \\
\text { coated stem } / 34 \\
\text { uncoated) }\end{array}$ & 69 & $27(24-50)$ & $\begin{array}{l}\text { Coated/uncoated: } \\
\text { ASES 94/83, } \\
\text { SANE 94/34 (no } \\
\text { significance) }\end{array}$ & $\begin{array}{l}\text { No loosening } / 1 \text { subsidence/ } 7 \\
\text { radiolucencies in coated } \\
\text { group } \\
1 \text { loosening/7 at risk of } \\
\text { loosening/ } 15 \text { radiolucencies } \\
\text { in uncoated group }\end{array}$ \\
\hline
\end{tabular}


Table 2. Outcomes in Reverse Total Shoulder Arthroplasty

\begin{tabular}{|c|c|c|c|c|c|c|}
\hline Study (year) & Implant & No. of patient & Age (yr) & $\mathrm{F} / \mathrm{U}(\mathrm{mo})$ & Outcome (score) & Complication \\
\hline $\begin{array}{l}\text { Atoun et al. }{ }^{15)} \\
\quad(2014)\end{array}$ & Verso (Biomet) & 31 & $74(58-93)$ & $36(24-52)$ & Constant 56 & $\begin{array}{l}\text { No humeral loosening or subsidence } \\
2 \text { glenoid notching } \\
3 \text { intraoperative fractures } \\
5 \text { periprosthetic fracture }\end{array}$ \\
\hline $\begin{array}{l}\text { Giuseffi et al. }^{14)} \\
\text { (2014) }\end{array}$ & Biomet (Mini) & 44 & $76(56-92)$ & $27(24-40)$ & $\begin{array}{l}\text { Mild or no pain (98\%), Neer } \\
\text { Score, excellent or satisfactory } \\
(95 \%)\end{array}$ & $\begin{array}{l}\text { Malalignment in } 1 \text { patient, no } \\
\text { humeral loosening }\end{array}$ \\
\hline $\begin{array}{l}\text { Cisneros et al. }{ }^{9)} \\
(2016)\end{array}$ & Verso (Biomet) & 98 & $74(38-93)$ & $\begin{array}{l}\text { Minimum } 2 \\
\text { years } F / U \\
(2-7 \text { years })\end{array}$ & Constant (adjusted) 59, SSV 85 & $\begin{array}{l}\text { No radiolucencies, subsidence or } \\
\text { stress shielding } \\
\text { Glenoid notching in } 21 \text { patients }\end{array}$ \\
\hline
\end{tabular}

Values are presented as number only or median (range).

F/U: follow-up, SSV: subjective shoulder value.

24-month follow-up in 49 patients, showing a favorable clinical outcome. Giuseffi et al. ${ }^{14)}$ found that reverse arthroplasty through the Mini humeral component effectively resolved pain and was not associated with stem loosening.

\section{2) Arthrex}

To the best of our knowledge, there are no clinical studies on Univers Apex (Arthrex, Naples, FL, USA) published to date, and it is not currently in use in Korea.

\section{3) Tornier (Wright)}

One of the few products available in Korea is the Aequalis Ascend Flex (Tornier, Edina, MN, USA). In at least a 24-month follow-up, Casagrande et al. ${ }^{17)}$ found that, among 73 patients, 4 required a revision arthroplasty and 5 patients had humeral loosening ( 1 for aseptic loosening and 4 for infection); overall, however, most patients showed a fair clinical outcome. In particular, a relatively high rate of radiolucency was observed surrounding the stem (71\%). The prosthetic implants used in this study were an earlier version without proximal porous coating. In a later study, Morwood et al. ${ }^{18)}$ who used an upgraded version of the Aquelis Ascend system to compare stems with proximal porous coating $(n=34)$ and those without $(n=34)$ found that stem loosening was not observed in any patients and radiolucency was observed in 7 patients (20.6\%) in the former group whilst there were 1 case (2.9\%) of aseptic loosening, 7 cases of $(20.6 \%)$ at risk of loosening, and 15 cases (44.1\%) of radiolucency in the latter group. Therefore, their report suggested that proximal porous coating in short-stem humeral components can lower stemrelated complications.

\section{Conclusion}

In sum, we found that short-stem humeral components in shoulder arthroplasty, initially developed to overcome limitations of long humeral stems, leads to fair clinical outcomes across different brands and designs of the prosthetic implants, for instance, whether the prostheses is designed for an anatomic or reverse shoulder arthroplasty. However, because there are clear drawbacks of short humeral stems that physicians may experience and there is a clear lack of long-term and large population studies on short humeral stems, there is a need for researchers to investigate the long-term clinical significance of short-stem humeral components and their advantages.

\section{References}

1. Cil A, Veillette CJ, Sanchez-Sotelo J, Sperling JW, Schleck C, Cofield RH. Revision of the humeral component for aseptic loosening in arthroplasty of the shoulder. J Bone Joint Surg Br. 2009;91(1):75-81.

2. Zumstein MA, Pinedo M, Old J, Boileau P. Problems, complications, reoperations, and revisions in reverse total shoulder arthroplasty: a systematic review. J Shoulder Elbow Surg. 2011;20(1):146-57.

3. Athwal GS, Sperling JW, Rispoli DM, Cofield RH. Periprosthetic humeral fractures during shoulder arthroplasty. J Bone Joint Surg Am. 2009;91(3):594-603.

4. Bohsali KI, Wirth MA, Rockwood CA Jr. Complications of total shoulder arthroplasty. J Bone Joint Surg Am. 2006;88(10): 2279-92.

5. Chin PY, Sperling JW, Cofield RH, Schleck C. Complications of total shoulder arthroplasty: are they fewer or different? J Shoulder Elbow Surg. 2006;15(1):19-22.

6. Farng E, Zingmond D, Krenek L, Soohoo NF. Factors predicting complication rates after primary shoulder arthroplasty. J Shoulder Elbow Surg. 2011;20(4):557-63.

7. Kumar S, Sperling JW, Haidukewych GH, Cofield RH. Periprosthetic humeral fractures after shoulder arthroplasty. J Bone Joint Surg Am. 2004;86(4):680-9.

8. Sperling JW, Cofield RH, Rowland CM. Minimum fifteen-year follow-up of Neer hemiarthroplasty and total shoulder arthroplasty in patients aged fifty years or younger. J Shoulder Elbow Surg. 2004;13(6):604-13. 
9. Cisneros LG, Atoun E, Abraham R, Tsvieli O, Bruguera J, Levy O. Revision shoulder arthroplasty: does the stem really matter? J Shoulder Elbow Surg. 2016;25(5):747-55.

10. Sahota S, Sperling JW, Cofield RH. Humeral windows and longitudinal splits for component removal in revision shoulder arthroplasty. J Shoulder Elbow Surg. 2014;23(10):1485-91.

11. Razfar N, Reeves JM, Langohr DG, Willing R, Athwal GS, Johnson JA. Comparison of proximal humeral bone stresses between stemless, short stem, and standard stem length: a finite element analysis. J Shoulder Elbow Surg. 2016;25(7):1076-83.

12. Raiss $P$, Edwards TB, Deutsch A, et al. Radiographic changes around humeral components in shoulder arthroplasty. J Bone Joint Surg Am. 2014;96(7):e54.

13. Lee M, Chebli C, Mounce D, Bertelsen A, Richardson M, Matsen $\mathrm{F}$ 3rd. Intramedullary reaming for press-fit fixation of a humeral component removes cortical bone asymmetrically. J Shoulder Elbow Surg. 2008;17(1):150-5.

14. Giuseffi SA, Streubel P, Sperling J, Sanchez-Sotelo J. Short-stem uncemented primary reverse shoulder arthroplasty: clinical and radiological outcomes. Bone Joint J. 2014;96(4):526-9.

15. Atoun E, Van Tongel A, Hous N, et al. Reverse shoulder arthroplasty with a short metaphyseal humeral stem. Int Orthop. 2014;38(6):1213-8.

16. Jost PW, Dines JS, Griffith MH, Angel M, Altchek DW, Dines DM. Total shoulder arthroplasty utilizing mini-stem humeral components: technique and short-term results. HSS J. 2011; 7(3):213-7.

17. Casagrande DJ, Parks DL, Torngren T, et al. Radiographic evaluation of short-stem press-fit total shoulder arthroplasty: shortterm follow-up. J Shoulder Elbow Surg. 2016;25(7):1163-9.

18. Morwood MP, Johnston PS, Garrigues GE. Proximal ingrowth coating decreases risk of loosening following uncemented shoulder arthroplasty using mini-stem humeral components and lesser tuberosity osteotomy. J Shoulder Elbow Surg. 2017; 26(7):1246-52. 